\title{
Utilização de novas tecnologias na popularização da língua brasileira de sinais
}

\author{
Carlos Henrique de Araújo Monteiro \\ Centro Federal de Educação Tecnológica Celso Suckow da Fonseca - CEFET/RJ \\ focus.carloshenriquemonteiro@gmail.com \\ Luiz Felipe Inácio Leite Pecoraro \\ Centro Federal de Educação Tecnológica Celso Suckow da Fonseca - CEFET/RJ \\ luizfelipeinacio@gmail.com \\ Anna Regina Corbo \\ Centro Federal de Educação Tecnológica Celso Suckow da Fonseca - CEFET/RJ \\ annarcc@gmail.com
}

\section{Resumo}

O presente artigo contém propostas de ações de extensão que estão sendo implementadas com a finalidade de divulgar a Língua Brasileira de Sinais (LIBRAS) numa comunidade preponderamente não surda da região metropolitana do Rio de Janeiro. Tais propostas analisam a relação existente entre a ausência de informação sobre a pedagogia inclusiva do surdo e a escassez de políticas públicas que implementam medidas de inclusão social. Com realizações de sessões de captura de vídeo de gestos, foi possível ensinar gestos em LIBRAS para sujeitos ouvintes, além de promover debates sobre temas relativos à surdez e à comunicação. Desse modo, foi possível alcançar a principal meta deste trabalho: alertar aos voluntários sobre o abismo linguístico existente entre surdos e ouvintes. Ao total, no ano de 2012, foram ensinados 24 verbetes em LIBRAS para 57 voluntários, e três instituições públicas estiveram envolvidas na realização deste.

Palavras-chave: LIBRAS. Surdez. Inclusão social. Novas tecnologias.

\section{Use of new technologies in dissemination of brazilian sign language}

\begin{abstract}
This paper contains proposals for university extension activities that are being implemented in order to disseminate Brazilian Sign Language (LIBRAS) in a community located in Rio de Janeiro's metropolitan area where most people are non-deaf. The proposals analyze the relationship between the lack of information about inclusive pedagogy for the Deaf and the lack of public policies to implement actions of social inclusion. Along the video recording sessions it was possible to teach LIBRAS gestures to hearing individuals and to promote discussions on topics related to deafness and communication. Thus it was possible to achieve the main goal of this work: to alert the volunteers about the linguistic abyss that exists between deaf and hearing persons. Along the year of 2012, a total of
\end{abstract}


24 words in LIBRAS was taught to 57 volunteers at 3 public institutions involved in completing this project.

Keywords: LIBRAS. Deafness. Social inclusion. New technologies.

\section{INTRODUÇÃO}

Sabe-se que o processo de interação social entre sujeitos surdos e ouvintes não ocorre de modo integral, sendo este um tipo de relação em níveis socialmente insatisfatórios. Em geral, a inclusão de surdos em nossa sociedade se dá pela adaptação, em mão única, ao que é considerado normal: a fala e a escrita; já a adaptação da sociedade ouvinte raramente ocorre.

Segundo Skliar (1998), a comunicação entre surdos e ouvintes é um meio fundamental de inclusão social. No entanto, a sociedade costuma tratar o assunto de maneira artificial, incluindo o aluno surdo pelo discurso da surdez como incapacidade, caracterizando assim o ouvintismo ${ }^{1}$ curricular no qual se defende a cura da surdez através de terapias de fala.

Diversas pesquisas referentes à cultura surda explicitam que o processo educacional brasileiro normalmente forma obstáculos que impedem a inserção da comunidade surda em diversos equipamentos do Estado (DÍAS; ROCHA, 2011). Do ponto de vista prático, deve-se realizar campanhas educacionais a fim de esclarecer a sociedade sobre essa problemática e tornar igualitário o acesso de surdos em diversos setores sociais.

Um importante fato que aconteceu na década passada foi a promulgação da Lei no 10.436 , de 24 de abril de 2002, que reconheceu a LIBRAS como um meio legal de comunicação e expressão. Nesse contexto, percebe-se que a visibilidade dos surdos acentuou-se, e, por conseguinte, estes vêm cada vez mais sendo relatados em vários artigos sobre inclusão educacional e social, como em Basso (2003), Quadros (2003), Sole (2010) e Thoma et al. (2010).

Dentro desse contexto, este artigo se propõe a relatar ações de extensão com a finalidade de divulgar a Língua Brasileira de Sinais (LIBRAS) dentro de uma comunidade escolar preponderantemente ouvinte e discutir novas alternativas para inclusão de surdos na sociedade. Essas ações aconteceram dentro do projeto "Reconhecimento Digital de Gestos em LIBRAS: Uso da Tecnologia para o Estreitamento da Comunicação entre Ouvintes e Surdos", desenvolvido pelos autores no CEFET/RJ, na Unidade de Ensino de Nova Iguaçu (CEFET/RJ UnED NI).

Este projeto é desenvolvido em duas grandes linhas: a) a linha de pesquisa, que consiste em desenvolver um programa computacional capaz de identificar gestos em LIBRAS. Esse protótipo, que atualmente está em fase intermediária de desenvolvimento, consiste em um tradutor digital de LIBRAS, seguindo o modelo de captação e classificação de amostras de vídeos de Dias et al. (2006) e Ong e Ranganath (2005), porém com modificações na metodologia; b) a linha de extensão, onde foram realizados encontros com a comunidade com o propósito de promover discussões e esclarecer o público-alvo sobre as diversas limitações que os surdos encontram no convívio em sociedade. Nos encontros, foram explicadas aos voluntários as vantagens que poderão ser obtidas quando existir um programa computacional eficiente capaz de interpretar gestos em LIBRAS.

Durante essas sessões de extensão, ensinamos aos voluntários um conjunto de palavras em LIBRAS, que foram previamente selecionadas através da análise do Dicionário da Língua Brasileira de Sinais (INES, 2005), e posteriormente iniciamos as gravações dessas palavras utilizando uma webcam 1 ouvintismo: segundo Skliar (1998, p. 15), "é um conjunto de representações dos ouvintes, a partir do qual o surdo está obrigado a olhar-se e narrar-se como se fosse ouvinte". 
com o intuito de produzir um banco de dados para testar o programa computacional.

Desse modo, o objetivo principal foi divulgar a cultura surda, promover a interação entre ouvintes e surdos e criar uma interface para que a plataforma de identificação de LIBRAS seja efetivamente testada, debatida e aprimorada junto com a comunidade interna e externa à nossa instituição.

\section{Material e Métodos}

O projeto foi realizado na Unidade de Ensino Descentralizada de Nova Iguaçu do Centro Federal de Educação Tecnológica Celso Suckow da Fonseca (CEFET-RJ UnED Nova Iguaçu). Esta pesquisa recebe o apoio institucional do Instituto Nacional de Educação de Surdos (INES) e da Secretaria Municipal de Pessoas com Deficiência (Semdpedef) da Prefeitura Municipal de Nova Iguaçu. No escopo deste projeto, pretende-se formar outras parcerias, a fim de discutir, planejar e executar ações de cunho social.

Inicialmente, uma das dificuldades encontradas foi a ausência de referências culturais sobre a LIBRAS. Para contornar esse problema, recebemos o apoio do INES, que forneceu um kit composto por materiais educacionais, artigos e demais documentos que relatam a experiência com surdos.

As áreas de atuação deste projeto estão direcionadas não só para promover a inclusão social através das ações de extensão, mas também para desenvolver um software fundamentado na Matemática, na Visão Computacional e no Processamento de Imagens. Na Ilustração 1, é descrita a esquematização das principais áreas de atuação do projeto:

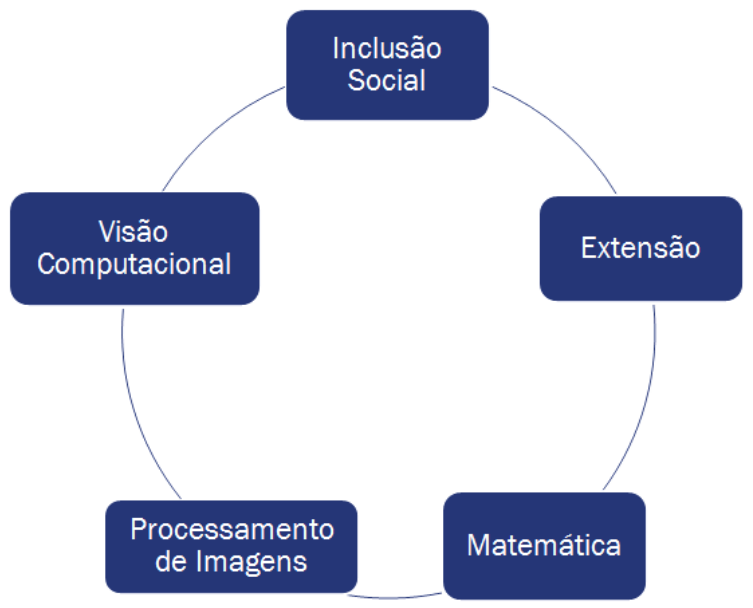

Ilustração 1 - Áreas de atuação do projeto.

Inclusão Social: A pedagogia de inclusão do surdo indica que a aproximação de sujeitos surdos e ouvintes deve ser estimulada em diversos segmentos sociais. Os envolvidos nesta comunicação podem desenvolver-se cognitivamente, fisicamente e aprimorar os conhecimentos.

Extensão: A Declaração Universal dos Direitos Humanos manifesta o princípio da não discriminação e explicita que todo indivíduo possui direito à educação. De acordo com essa vertente, o projeto expõe, nos debates realizados nas sessões, que os surdos devem ser incluídos no processo educacional com a finalidade de preservar sua cidadania.

Matemática: Esta ciência exata possui uma ampla aplicabilidade no processo de elaboração do programa que detecta os gestos da LIBRAS. Utiliza-se um embasamento teórico que faz referência a subáreas como Álgebra Linear, Ánálise e Estatística com o propósito de elevar a eficiência do software.

Processamento de Imagens: Este ramo de pesquisa científica fornece o embasamento técnico para produzir algoritmos computacionais capazes de extrair informações das imagens. Com o processamento de 
imagens é possível implementar códigos que minimizam distorções e degradações de imagens e identificam regiões de pixels.

Visão Computacional: As pesquisas elaboradas nesta área normalmente são destinadas a monitorar e orientar sistemas que exigem um elevado grau de precisão. Nesse caso, são utilizadas as aplicações baseadas no princípio de rastreamento de trajetória.

As ações de extensão deste projeto buscaram auxiliar no desenvolvimento do algoritmo de detecção de LIBRAS através da geração de um banco de dados. Além disso, foi proposta, simultaneamente, a divulgação da cultura surda. Para auxiliar esse processo foram colocadas em prática três linhas de trabalho: a) as sessões de captura de vídeos; b) a elaboração de um website; e c) a realização de palestras de divulgação.

\section{a) Sessões de captura de vídeos}

Inicialmente divulgamos o projeto no grupo de e-mails de discentes do CEFET-RJ UnED Nova Iguaçu, com o intuito de criar um quantitativo de voluntários e alocar adequadamente os recursos técnicos do projeto. Os materiais básicos utilizados durante a execução das filmagens foram: notebook, webcam e planos de fundo.

Os vídeos capturados pela webcam são dispostos em um formato que, devido à compressão, pode influenciar a taxa de identificação do programa computacional que está sendo implementado. Gravou-se cada palavra gesticulada pelos voluntários em três diferentes planos de fundo (branco, madeira e estante de livros) para mensurar os efeitos desta interferência. A Ilustração 2 mostra dois voluntários gesticulando palavras em LIBRAS durante uma das sessões de captura, em planos de fundo diferentes.

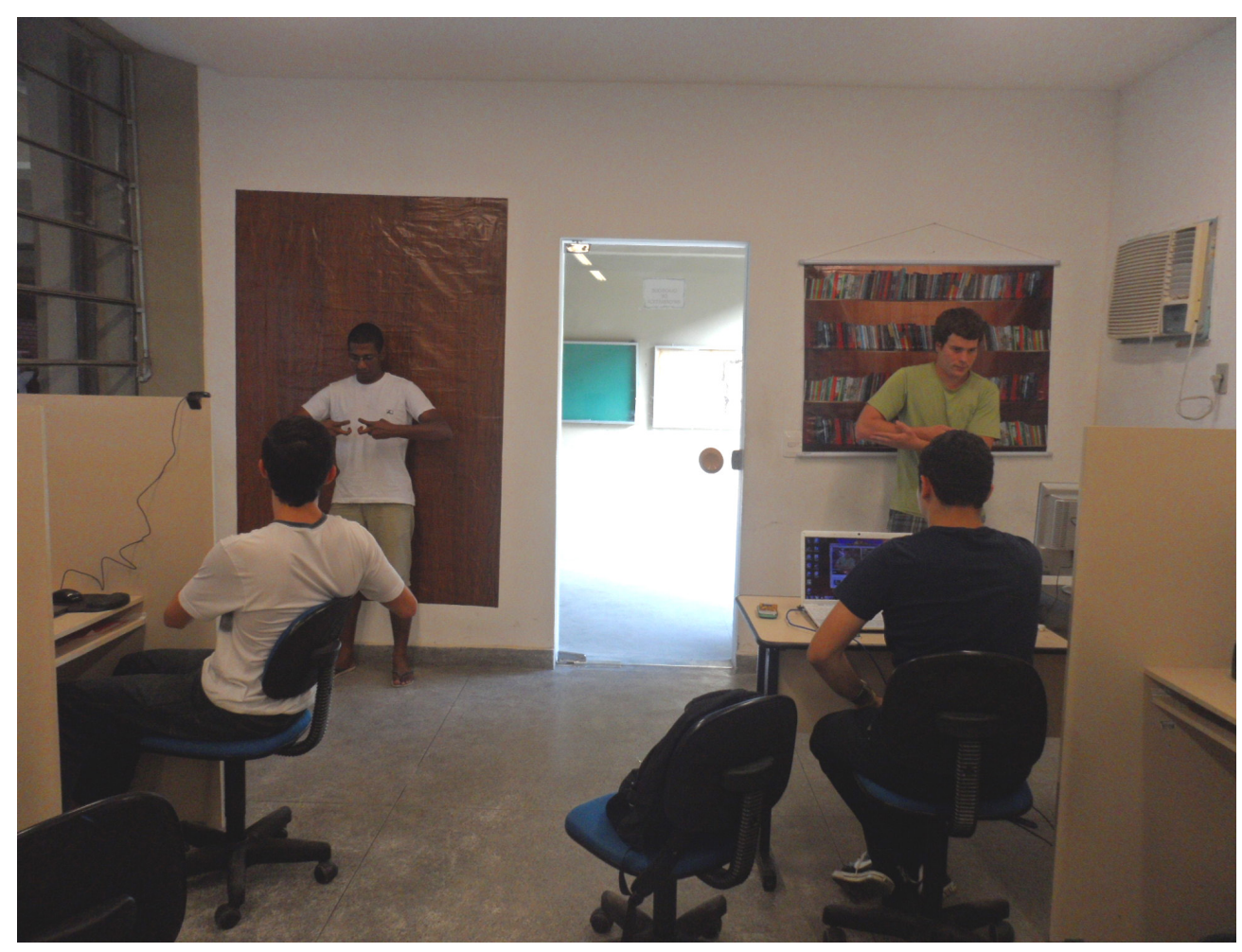

Ilustração 2 - Voluntários gesticulando diante de planos de fundo madeira e estante.

Os 24 tipos de sinais em LIBRAS utilizados para este projeto passaram por dois critérios de seleção: o primeiro critério define que as palavras não podem ser verbos, e o segundo divide as palavras em dois grupos de 12 palavras que conservam o padrão de movimentação das mãos segundo uma trajetória estrita- 
mente vertical ou horizontal.

Obanco de dados vertical é composto pelas palavras: andaime, depressão, fantasma, martelo, nacionalidade, neve, paciência, parede, redação, relâmpago, trampolim e vapor. Por outro lado, o banco de dados horizontal contém as palavras: amante, bebê, compromisso, dado, elástico, impossível, milagre, notícia, orquestra, surf, televisão e veloz.

Durante as sessões de gravação, foram exibidos para os voluntários os vídeos de referência de cada palavra, a fim de ensiná-los a correta representação dos sinais. Alertou-se também os participantes sobre a forma ideal de executar os sinais diante da webcam objetivando minimizar os erros que são cometidos devido à ultrapassagem dos limites de gravação da câmera.

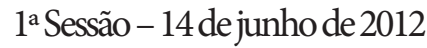

Ocorreu no CEFET-RJ UnED Nova Iguaçu, e o principal destaque foi a elevada participação de voluntários, pois nesse dia essa instituição sediou o II Encontro Regional de Engenharia e Desenvolvimento Social (EREDES). No total, 20 pessoas participaram das gravações, destas, 11 são homens e 9 são mulheres.

\section{$2^{a}$ Sessão - 14 de junho de 2012}

Aconteceu no CEFET-RJ UnED Nova Iguaçu, e, devido ao período de greve, não foi possível obter um elevado número de participantes para esse evento. Registraram-se 11 participantes no total, sendo 6 homens e 5 mulheres.

\section{$3^{a}$ Sessão - 14 de setembro de 2012}

Essa sessão de extensão foi realizada em parceria com a Prefeitura Municipal de Nova Iguaçu, no auditório do Sindicato dos Químicos de Nova Iguaçu. Nesse evento estiveram presentes os 5 alunos (entre eles 1 homem e 4 mulheres) do curso de LIBRAS que é oferecido pela prefeitura.

\section{$4^{a}$ Sessão - 26 e 29 de novembro de 2012}

Esse evento foi realizado no CEFET-RJ UnED Nova Iguaçu, contou com divulgação em redes sociais e, pela primeira vez, foi iniciada a aquisição de amostras do banco de dados de sinais horizontais. Destaca-se a participação de um total de 21 pessoas, sendo 17 homens e 4 mulheres.

\section{b) Desenvolvimento do site}

A Internet normalmente facilita o acesso da população a conteúdos relacionados com a produção intelectual de materiais educativos. Com o objetivo de divulgar este projeto para um público maior e especificamente interessado em nossa temática, foi elaborado um website em um domínio gratuito que contém informações sobre o projeto e sobre o que vem sendo produzido com as atividades de extensão. A Ilustração 3 mostra a página inicial do site do projeto, cujo endereço eletrônico é: $<\underline{\text { http://sites.google.com/ }}$ site/projetolibrascefet/>.

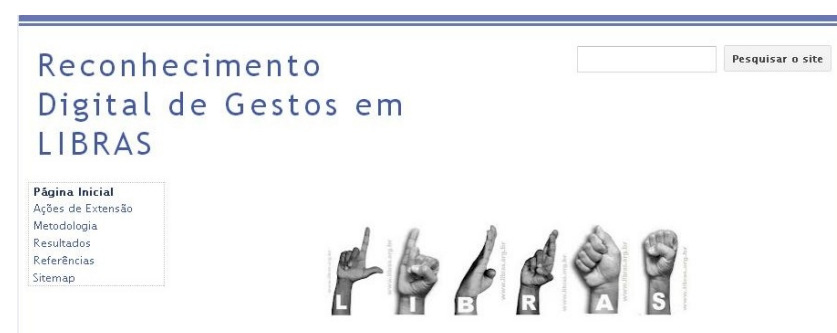

O projeto "Reconhecimento digital de gestos em LIERAS: uso da tecrologia para o estreitamento da comunicacão entre ouvintes e surdos" é coordenado pela professora Anna Regina Corbo Costae desenvolvido pelos bolsistas Carlos Henrique de Araúio Mon
Felipe Inacio Leite Pecorara (Bolsista PBEXT/CEFET-RJ).

O objetivo geral do projeto é a elaboração de un programa computacional que seja capaz de

interpretar os sinais em LIRRAS através de uma cămera digital. O intuito desta pesquisa é estimular

a comunicaçăo entre ouvintes e surdos, utilizando a tecnologia através de um identificador que, em

longo prazo, possa ser eficienternente testado em escolas públicas.

llustração 3 - Página inicial do website do projeto. 


\section{c) Palestra e outros meios de divulgação}

A Semana de Extensão é um evento que ocorre anualmente no CEFET-RJ UnED Nova Iguaçu no âmbito da Semana Nacional de Ciência e Tecnologia, cujo principal objetivo é expor para a sociedade os projetos que estão sendo desenvolvidos nessa instituição pública de ensino. No dia 18 de outubro de 2012, durante este evento, a equipe executora ministrou uma palestra explicitando o escopo extensionista deste projeto para um público externo.

Além disso, no dia 22 de setembro de 2012, o jornal “O Dia”, de circulação estadual, publicou uma nota informativa, na qual foi retratado o pioneirismo desse tipo pesquisa no município de Nova Iguaçu.

\section{RESULTADOS E ANÁLISE}

Urge a necessidade de desenvolver campanhas educacionais capazes de alertar a sociedade sobre os diversos aspectos do processo de interação social entre ouvintes e surdos. É importante promover projetos que divulguem a competência linguística da LIBRAS, a fim de conscientizar a sociedade e facilitar o acesso dos surdos aos aparelhos do Estado.

Durante as sessões de captura de vídeos deste projeto de extensão, percebeu-se que a cultura surda despertou um surpreendente interesse na comunidade acadêmica do CEFET-RJ UnED Nova Iguaçu. Destaca-se também que, durante os debates os voluntários, foram levantadas questões sobre a semântica dos sinais, relatos de históricos de dificuldade de comunicação, sugestões para aprimoramento nas relações interpessoais entre ouvintes e surdos, além de dúvidas quanto às técnicas que vêm sendo implementadas no software.

Claramente, o principal resultado obtido foi a possibilidade de debater com um total de 57 voluntários as ações inclusivas contidas nesta pesquisa. Durante as sessões, foi possível entender o ponto de vista de professores, alunos e intérpretes sobre as propostas contidas neste estudo e, além disso, produzir um total de 1.047 amostras de vídeos.

Enxerga-se também a necessidade de futuramente elevar o número de parceiros deste projeto e promover sessões de extensão com indivíduos surdos. Numa nova etapa, será imprescindível que seja registrada a opinião da comunidade de surdos não só sobre a implementação de um software capaz de ampliar o canal de comunicação, mas também sobre o panorama de ideias que está contido nesta pesquisa.

\section{Considerações Finais}

Permanece como obstáculo garantir que a hegemonia de culturas ouvintes não minimize a utilização da LIBRAS nas relações sociais. Entende-se que normalmente o processo educacional brasileiro não absorve e expõe para a sociedade a cultura surda fora do contexto separatista, por conseguinte ocorre a formação de uma lacuna na comunicação entre sujeitos ouvintes e surdos.

As principais atividades de extensão que estão sendo executadas neste projeto foram planejadas com a finalidade de divulgar a cultura surda e auxiliar na elaboração de um software detector de LIBRAS. Nas sessões de captura de vídeos, realizaram-se debates com o intuito de analisar os obstáculos que geralmente impedem os surdos de obterem amplo acesso à informação e ao convívio social.

De modo geral, o objetivo de promover a LIBRAS em uma comunidade preponderamente ouvinte foi atingido. Adicionalmente, o projeto também cumpriu a meta de se propor a discutir não só a integração dos surdos na sociedade, mas também de ascender às condições de acessibilidade para que este grupo possa interagir com as várias redes de informação. 


\section{REFERÊNCIAS}

BASSO, Idavania. Mídia e educação de surdos: transformações reais ou uma nova utopia?. Ponto de Vista, n.05, p. 113-128. Florianópolis: UFSC, 2003.

DIAS, J. B.; SOUZA, K. P. de; PISTORI, H. Conjunto de Treinamento para Algoritmos de Reconhecimento de LIBRAS. II Workshop de Visão Computacional, São Carlos, Outubro 16-18, 2006.

DÍAZ, Cora Maria de Oliveira Beleño; ROCHA, José Geraldo da. Políticas Públicas que Normatizam as Práticas Pedagógicas na Educação de Surdos. Espaço: Informativo Técnico-Científico do INES, Rio de Janeiro, n. 0130-7668, p.110-123. 2012. .

INES - Instituto Nacional de Educação de Surdos. Dicionário da Língua Brasileira de Sinais - LIBRAS , CD-ROM Versão 2.0, 2005.

ONG, S.C.W. \& RANGANATH, S. Automatic sign language analysis: A survey and the future beyond lexical meaning. IEEE Trans. Pattern Anal. Mach. Intell.,27(6):873-891, 2005.

QUADROS, Ronice. Situando as diferenças implicadas na educação de surdos: inclusão/exclusão. Ponto de Vista, n.05, p. 81-111. Florianópolis: UFSC, 2003.

SKLIAR, C. B. (Org.). A surdez: um olhar sobre as diferenças. Porto Alegre: Mediação, 1998.

SOLE, Maria Cristina Petrucci. Transtornos do desenvolvimento e a aprendizagem do sujeito surdo. Forum: Instituto Nacional de Educação de Surdos, Rio de Janeiro, v. 22, n. 1518-2509, p.15-24. 2010.

THOMA, Adriana; SCHUCK, Pedro ; RADUNZ, Maikel ; KOHN, Sabrina. UNISCriança: inclusão digital através de softwares com escrita de sinais. Forum: Instituto Nacional de Educação de Surdos, Rio de Janeiro, v. 22, n. 1518-2509, p.15-24. 2010. 